\title{
ASTROMETRY IN LOCAL REFERENCE FRAMES FOR DEEP SPACE NAVIGATION
}

\author{
R. N. Treuhaft \\ Jet Propulsion Laboratory, California Institute of Technology \\ 4800 Oak Grove Drive \\ Pasadena, California 91109
}

\begin{abstract}
Future deep space missions will benefit from submilliarcsecond angular navigation accuracy ( $<25 \mathrm{~km}$ projected distance at Neptune). Conventional Very Long Baseline Interferometry (VLBI) measurement strategies entail observations of a radio source (RS) and spacecraft pair. To increase the 3-5 milliarcsecond accuracies currently attainable, instrumental improvements could be coupled with either of two methods: 1) Use improved calibration measurements with the single RS as the spacecraft reference, or 2) use a reference frame established by a local network of RS's near the spacecraft to reduce the contribution of sky-dependent error sources. The demanding baseline vector (earth orientation) and tropospheric calibrations needed to realize submilliarcsecond accuracy with the single-source strategy would not be necessary with the multi-source strategy. The two strategies are compared via a sample covariance analysis. In this analysis, the single-source method yields 1.5 milliarcseconds for the navigation accuracy, while the multi-source method yields 0.5 milliarcseconds.
\end{abstract}

Deep space angular navigation is currently achieved with VLBI measurements of a spacecraft and a radio source (RS), typically within 10 degrees of each other. Demonstrated accuracies for this technique have been in the range of 3-5 milliarcseconds for the projection of the unmodelled spacecraft position on the baseline vector (J. S. Border et. al., 1982). Improvements in instrumentation in the Deep Space Network (DSN), particularly the increased bandwidth and bit rates attainable with the Mark III VLBI system (Rogers et. al., 1983), will allow rapid observations of a few RS's near the spacecraft. These additional RS observations could be used to calibrate errors which are a function of the direction in which the antennas point, called sky-dependent errors. The local reference frame technique described here is an extension of the technique used to measure relativistic bending (Fomalont and Sramek, 1975). Below, an example of a navigation covariance analysis is given, describing the parameterization of the sky-dependent errors, the observational configuration, and the results for the conventional and local network techniques.

The conventional and local network techniques can be viewed as estimations of an unmodelled spacecraft delay parameter in the presence of other unmodelled effects. With the conventional technique, assuming that the observation sequence is RS-spacecraft-RS, only station clock epoch and clock rate are estimated along with the desired spacecraft delay. All other errors which cause delay differences between the observations of the two objects propagate into the spacecraft delay; they will be called "consider" errors, as 
opposed to "explicit" errors, which are explicitly solved for in the estimation procedure. In the proposed local network technique, the two largest sky-dependent errors, earth orientation and tropospheric uncertainties, become explicit parameters. Therefore, to the extent that they are correctly modelled, the effects of earth orientation, troposphere, and clocks do not need to be externally calibrated.

Table 1 below describes the covariance results for the two navigation methods. The levels of assumed errors are shown along with the level at which they affect the spacecraft angular determination. The DSN California-Australia baseline $(\sim 10000 \mathrm{~km})$ was used with five radio sources from the DSN catalog forming the local net. With RS1 being radio source \#1, the local network technique entailed seven VLBI observations in the sequence RS1-RS2-RS3-spacecraft-RS4-RS5-RS1. For the conventional technique, the closest source, RS3, was assumed to be observed in the sequence RS3-spacecraft-RS3.

TABLE 1. Comparison of Conventional and Local Net Errors

\begin{tabular}{|c|c|c|c|}
\hline Error & Assumed Magnitude & $\begin{array}{c}\text { Conventional } \\
\text { Error }(\mu \mathrm{as})\end{array}$ & $\begin{array}{l}\text { Local Net } \\
\text { Error ( } \mu \text { as) }\end{array}$ \\
\hline Earth Orient. & $\begin{array}{l}10 \text { mas per } \\
\text { component }\end{array}$ & 1352 & explicit \\
\hline Static Trop. & $4 \mathrm{~cm}$ & 476 & explicit \\
\hline Fluctuating Trop. & $\begin{array}{l}1 \mathrm{~cm} \text { daily } \\
\text { zen/station }\end{array}$ & 299 & 180 \\
\hline Baseline Length & $3 \mathrm{~cm}$ & 79 & 10 \\
\hline Celestial Pole & $\begin{array}{l}5 \text { mas long. } \\
5 \text { mas obl. }\end{array}$ & 0.3 & 13 \\
\hline System Noise & $\begin{array}{c}30 \text { psec spacecraft } \\
30 \text { psec RS }\end{array}$ & 213 & 427 \\
\hline RSS & & 1482 & 464 \\
\hline
\end{tabular}

The results suggest that the local network technique is a promising alternative to conventional navigation, with minimal external calibration required. The earth orientation error, assumed at the approximate level of that required for the Galileo mission to Jupiter, dominates the conventional technique error for the RS-spacecraft configuration studied. It should be noted that the exact parameterization of earth orientation and troposphere effects in the local network technique is preliminary, and the contribution from system noise may be reduced by a more judicious parameter selection. In this analysis, sources were considered as perfectly known points; effects of source structure and position errors must be considered in future analyses.

The research described in this report was carried out by the Jet Propulsion Laboratory, California Institute of Technology, under a contract with NASA.

\section{REFERENCES}

Border, J. S., et. al., AIAA Paper \#82-1471, August 1982.

Fomalont, E. B., and Sramek, R. A., Astrophysical Journal, 199, 749, August 1975.

Rogers, A. E. E. et. al., Science 219, 51, January 1983. 